\title{
Determination of an Innovative Consistent Law for the Rheological Behavior of Polymer/Carbon Nanotubes Composites
}

\section{Frédéric Thiébaud}

Laboratoire d’Énergétique et de Mécanique Théorique et Appliquée, UHP-ESSTIN, Vandoeuvre lès Nancy, France Email: frederic.thiebaud@esstin.uhp-nancy.fr

Received December 29 ${ }^{\text {th }}$, 2010; revised January 12 ${ }^{\text {th }}, 2011$; accepted January $15^{\text {th }}, 2011$.

\begin{abstract}
An innovative constitutive model for the rheological behavior of the polymer/carbon nanotubes composites is proposed in this paper. Based on experimental investigations, this consistent law gives the evolution of the composite shear viscosity versus the shear rate, over a large range, and the temperature with various carbon nanotubes mass fractions. Hence, this consistent could be implemented in a finite element code in order to lead many polymer/carbon nanotubes manufacturing process like injection molding or hot embossing.
\end{abstract}

Keywords: Carbon Nanotubes, Composite, Rheological Behavior

\section{Introduction}

Since their official discvovery in 1991 by Iijima [1], Carbon nanotubes (CNT) growing interest in the field of engineering. For example, their mechanical and physical properties make CNT some attractive candidates for use in composites (polymer/CNT) material applications.

These mechanical performances strongly depend on the composite melt process. Many studies have been carried out by several authors and referenced by Coleman et al. [2] and Breuer and Sundaraj [3]. Actually, many process such as injection molding, hot embossing, compacting, extrusion, etc., permit to realize high performances composite parts with CNT reinforcement. Hence, in order to optimize the process parameters and finally the performances of the so realized polymer/CNT composite, many investigations on the numerical simulation by the finite element method of theses process are leaded as usual.

The main difficulty to obtain some accurate results from these numerical simulations is to consider a consistent law for the rheological behavior of the polymer/CNT composite. A few studies are reported on the rheological properties of polymer/CNT prepared by melt mixing process. Teng et al. [4] investigated the effect of MWCNT content on the rheological properties of polypropylene at high shear rates $\left(10^{2}\right.$ to $\left.10^{4} \mathrm{~s}^{-1}\right)$ and a tem- perature around $200^{\circ} \mathrm{C}$. Thus, the authors proposed a power law as a constitutive model to characterize the rheological behavior of the composite but, this constitutive model doesn't take into account the Newtonian plateau at low shear rates. A similar study leaded by Seo et al. [5] focus on the rheological behavior of polypropylene/MWCNT composite at low shear rates $\left(10^{-1}\right.$ to $10^{2}$ $\mathrm{s}^{-1}$ ) at a temperature about $170^{\circ} \mathrm{C}$. A more convenient model proposed by Cross [5] allows switching from the Newtonian plateau at low shear rates to a straight line at high shear rates. However, this transition leads to a slope break that the model doesn't adjust.

Consequently, one notice that it is difficult to find a consistent law for the rheological behavior of the polymer/CNT composite on a very large shear rate range (typically $10^{-1}$ to $2 \times 10^{4} \mathrm{~s}^{-1}$ ) by considering the temperature effects, which are strongly present in the composites/CNT process.

\section{Experimental Investigations}

The aim of this work is to propose a constitutive model for the rheological behavior of the polymer/multi- walled carbon nanotubes (MWCNT) composite on a shear rate range (typically $10^{-1}$ to $2 \times 10^{4} \mathrm{~s}^{-1}$ ), with various MWCNT mass fractions $f$ usually considered in the engineering fields $(f=1,2,4$ and 8 wt\%), by considering the 
temperature effects. This constitutive model could be used in a finite element code to predict the fluid flow of the composite/MWCNT for major numerical simulations of composite/MWCNT process.

To do this, three steps are followed as well. In the first one, the production of the composites polymer/MWCNT with various MWCNT mass fractions was carried out. In the second one, the rheological behavior of the neat polymer and the polymer/MWCNT composites was investigated by using a capillary rheometer. Finally, these investigations allow identifying the rheological behavior for each polymer/MWCNT composite and the neat polymer.

Multi-Walled Carbon Nanotubes (MWCNT) were produced by Chemical Vapor Deposition (CVD) process. Their purity is more than $95 \%$ and one considers that their diameter is around $10-20 \mathrm{~nm}$, their length is around $1-25 \mu \mathrm{m}$ and their density is evaluated as $\rho_{c}=1.30 \mathrm{~g} / \mathrm{cm}^{3}$, according to the specifications of the provider. The polymer used in this work is polypropylene (PP) which is one of the most commonly used thermoplastic polymers. In pellets form, it is a PP1120 type that corresponds to a homopolymer with a Melt Flow Indice (MFI) around 15 $\mathrm{g} / 10 \mathrm{~min}\left(230^{\circ} \mathrm{C}, 2.16 \mathrm{~kg}\right)$. This PP grade is typically dedicated to injection molding and its density is evaluated as $\rho_{p}$ is equal to $0.90 \mathrm{~g} / \mathrm{cm}^{3}$.

Polypropylene was mixed with various MWCNT mass fractions $f(f=1,2,4$ and $8 \mathrm{wt} \%$ ) following the protocol described by Pötschke et al. [7], with a Brabender ${ }^{\circledR}$ mixer (Plastograph EC W50EHT). The temperature in the mixing cavities and the rotation speed of the screw are regulated around $200^{\circ} \mathrm{C}$ and $60 \mathrm{rpm}$ respectively, and the processing time is about $15 \mathrm{~min}$. Thus, bulk samples are collected and crushed into small particles in a grinder as it is shown in the Figure 1.

The shear viscosity is thus measured on a very large shear rate range: $\left(10^{-1}\right.$ to $\left.2 \times 10^{4} \mathrm{~s}^{-1}\right)$ at four temperatures $\left(180,200,220\right.$ and $\left.240^{\circ} \mathrm{C}\right)$. A Rosand ${ }^{\circledR}$ RH2000 capillary rheometer with two dies was used for these measurments. The first die $(\varnothing 1 \mathrm{~mm})$ was used in the shear range form $10^{-1}$ to $20 \mathrm{~s}^{-1}$ shear rate and the second die $(\varnothing 2 \mathrm{~mm})$ was used in the shear rate range 20 to $2 \times 10^{4} \mathrm{~s}^{-1}$. For example, the shear viscosity versus the shear rate for the neat PP and the fourth PP/MWCNT composites at $200^{\circ} \mathrm{C}$, are reputed in the Figure 2. Similarly the test temperature influence on the shear viscosity is shown in the Figure 3 for the $4 \mathrm{wt} \%$ composite. One assume here that the same pattern of results is identifiable to the other temperatures and the other composites (neat PP and 1, 2 and 8 wt\% PP/MWCNT composites).

At fixed temperature, the shear viscosity of the neat PP and PP/ MWCNT composites decreases with the increase of the shear rate. These viscosity curves clearly indicate the significant effect of the MWCNT content. When the shear rate is in the range $10^{-1}-10^{2} \mathrm{~s}^{-1}$, the PP/MWCNT composite exhibit a shear thinning effect whereas the neat PP exhibits only small shear rate dependence, revealing a Newtonian plateau. However, from 1 wt\% MWCNT content, the viscosity curves present a much steeper slope at low shear rate, and there is no Newtonian plateau within the shear rate range studied. Also, from 4 wt\% MWCNT, the viscosity curves appear nearly power law over this range of shear rate. This phenomenon is related to the fact that, the MWCNT-MWCNT interactions begin to dominate as the MWCNT content increases, eventually leading to a percolation and a formation of interconnected MWCNT structures. However, when the shear rate in the range $10^{2}$ to $2 \times 10^{4} \mathrm{~s}^{-1}$, the shear viscosity of the PP/MWCNT composite of the same order than the neat PP: the effect of the MWCNT content is less important than at low shear rates. When shear rate is increasing, the shear thinning effect is enhanced and the wall slip phenomena occurring between $\mathrm{PP} / \mathrm{MWCNT}$ and the wall of the rheometer is predominant, as it is supposed by Lee et al. [8] and Lam et al. [9]. Finally for one PP/MWCNT composite shade, the viscosity curves indicate a decrease of the shear viscosity with the increase of the temperature. This is due to the fact that the shear viscosity of the polypropylene decreases when heating.

\section{Rheological Behavior}

The previous investigations on the rheological behavior of the neat PP and the fourth PP/MWCNT composites clearly show a high dependence of the shear viscosity on

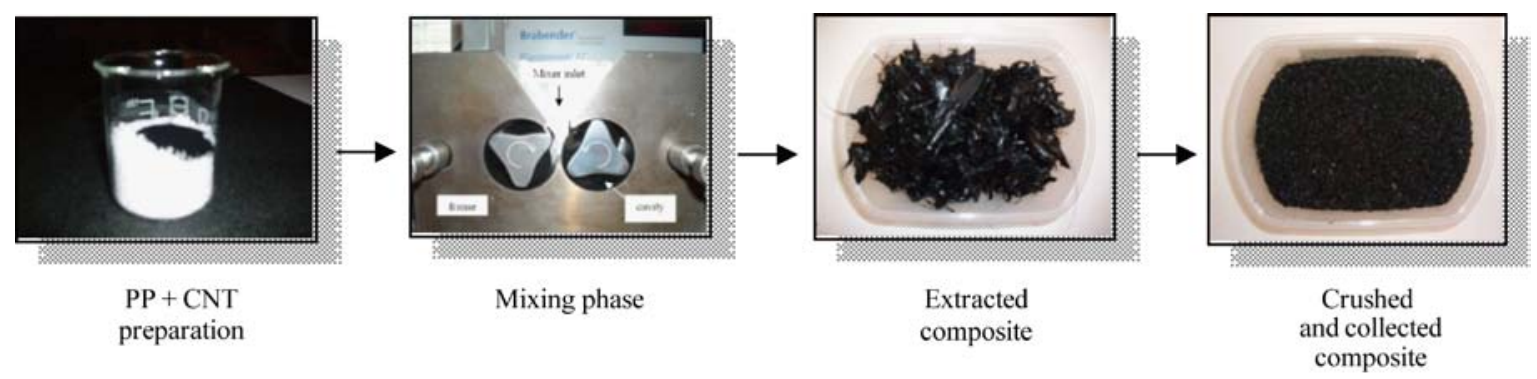

Figure 1. Manufacturing phase of the PP/MWCNT composite. 


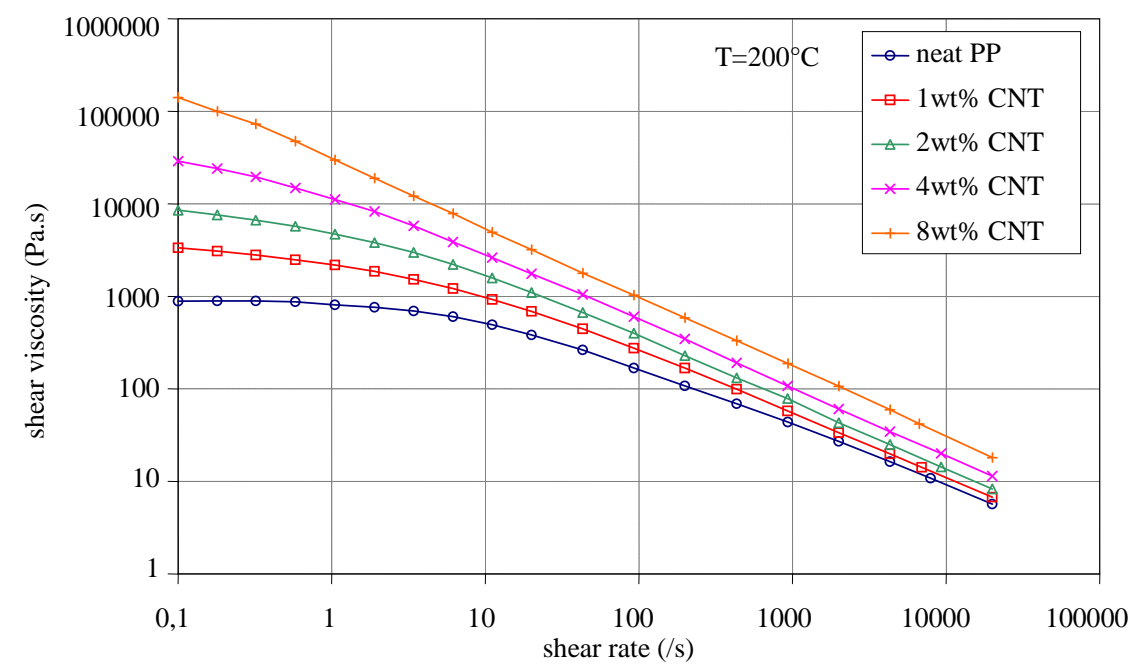

Figure 2. Shear viscosity vs shear rate at $200^{\circ} \mathrm{C}$ for the neat $\mathrm{PP}$ and the loaded 4 composites.

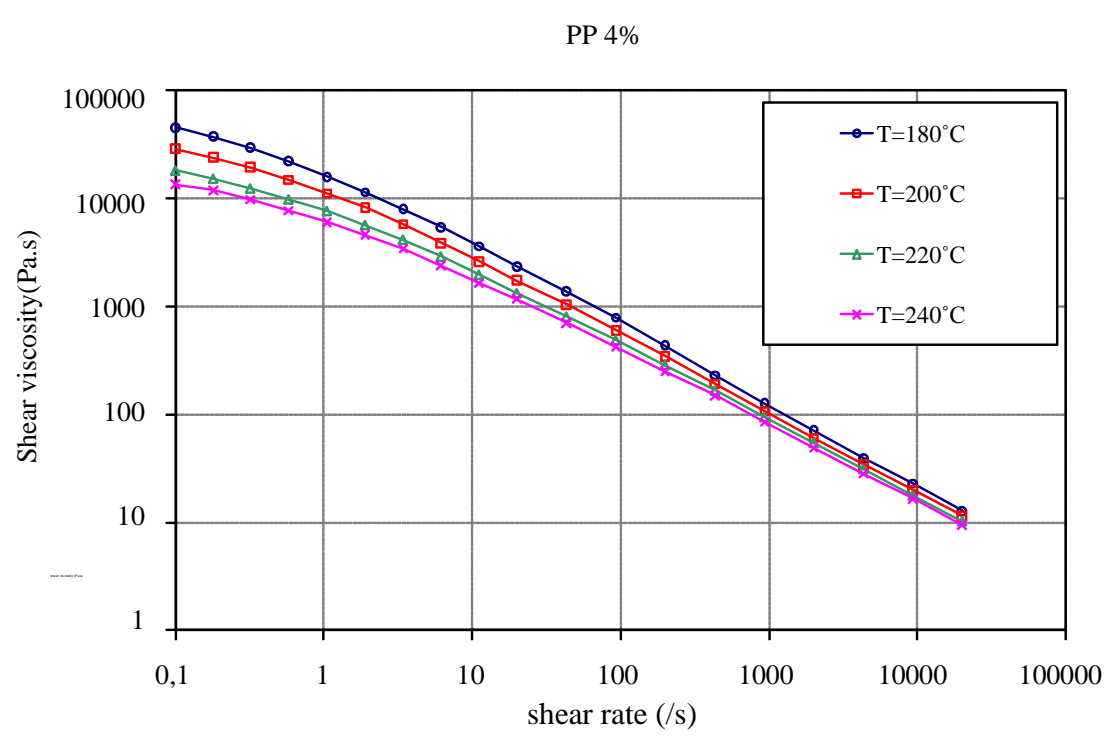

Figure 3. Shear viscosity vs shear rate at $180,200,220$ and $240^{\circ} \mathrm{C}$ for the $4 \mathrm{wt} \% \mathrm{PP} / \mathrm{MWCNT}$ composite.

the shear rate and the temperature. Thus, the constitutive model must be able to predict the flow of material under various processing conditions and particularly the shear rate and the temperature. Carreau [10] proposed a model to modify the slope break by changing the Cross law without adding additional parameters. This Carreau law expression is related in equation (1):

$$
\mu(\dot{\bar{\gamma}}, T)=\mu_{0}(T)\left(1+(\lambda \dot{\bar{\gamma}})^{2}\right)^{\frac{n-1}{2}}
$$

where $\mu(\dot{\bar{\gamma}}, T)$ is the shear viscosity, $T$ the temperature and $\dot{\bar{\gamma}}$ the equivalent shear rate defined by:

$$
\dot{\bar{\gamma}}=\sqrt{\frac{2}{3} \operatorname{dev}(\underline{\dot{\varepsilon}}): \operatorname{dev}(\underline{\dot{\varepsilon}})}
$$

with $\dot{\dot{\varepsilon}}$, defined as the shear rate tensor versus the velocity field $\mathbf{V}$ :

$$
\underline{\dot{\varepsilon}}=\frac{1}{2}\left(\underline{\operatorname{grad}}(\mathbf{V})+\underline{\operatorname{grad}}^{T}(\mathbf{V})\right)
$$

where $\operatorname{grad}(\bullet)$ is the well known gradient operator. $\operatorname{dev}(\underline{\dot{\varepsilon}})$, the deviatoric part of the shear rate tensor:

$$
\operatorname{dev}(\underline{\dot{\varepsilon}})=\underline{\dot{\varepsilon}}-\frac{1}{3} \operatorname{Tr}(\underline{\dot{\varepsilon}}) \underline{I}
$$

where $\underline{I}$ is the second order identity tensor and $\operatorname{Tr}(\bullet)$ the trace operator $\left(\operatorname{Tr}(A)=\mathrm{A}_{\mathrm{ii}}\right)$. The index $n$ is typically called the shear thinning index. The term $\mu_{0}(T)$ is identified as the material consistency and $\lambda$ is a characteristic relaxation time of the material. 


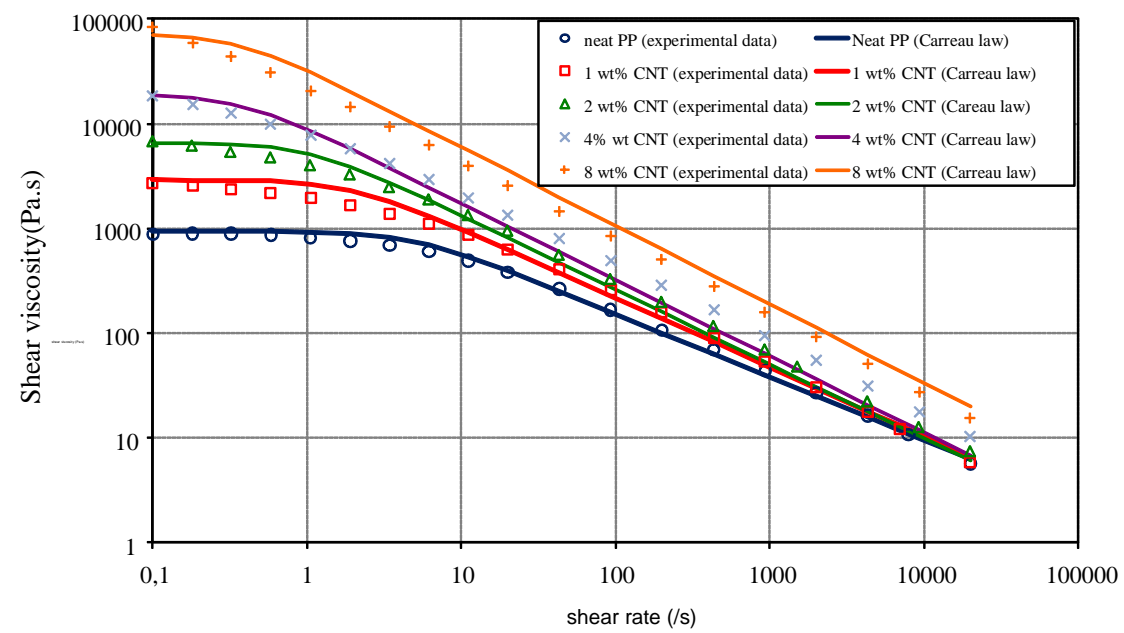

Figure 4. Shear viscosity as a function of the shear rate at 180, 200, 220 and $240^{\circ} \mathrm{C}$ for the 4 wt $\%$ PP/MWCNT composite: experimental data and Carreau law enriched temperature fitting curves.

Table 1. Rheological parameters for the neat $P P$ and each PP/MWCNT composite.

\begin{tabular}{cccccc}
\hline $\begin{array}{c}\text { MWCNT } \\
\text { content }\end{array}$ & $0 \mathrm{wt} \%$ & $1 \mathrm{wt} \%$ & $2 \mathrm{wt} \%$ & $4 \mathrm{wt} \%$ & $8 \mathrm{wt} \%$ \\
\hline$\mu_{0}\left(\mathrm{~Pa} \cdot \mathrm{s}^{\mathrm{n}}\right)$ & 20778 & 34214 & 172783 & $2 \cdot 10^{6}$ & $2 \cdot 10^{7}$ \\
$b\left(\mathrm{C}^{-1}\right)$ & 0.0155 & 0.0116 & 0.0148 & 0.0206 & 0.0242 \\
$\lambda\left(\mathrm{s}^{-1}\right)$ & 0.207 & 0.514 & 0.942 & 2.645 & 2.733 \\
$n$ & 0.4 & 0.34 & 0.29 & 0.27 & 0.25 \\
\hline
\end{tabular}

The thermal dependence is here modeled by the proposed expression for the material consistency:

$$
\mu_{0}(T)=\mu_{0} e^{-b T}
$$

where $b$ is another characteristic time of the material to be identified. With this suggested constitutive model, thermal effects are accounted and the transition from the power law to the Newtonian plateau is a more suitable.

The rheological parameters $\left(\mu_{0}, b, \lambda\right.$ and $\left.n\right)$ are summarized in Table 1 for the neat PP and each PP/ MWCNT composite. One can notice that the parameter $\mu_{0}$ increases with MWCNTs content while the shear thinning index $n$ substantially decreases. For the PP/MWCNT composite with 4 and 8 wt\% MWCNTs content, the parameter $\mu_{0}$ value is higher than for other contents. In these conditions, the constitutive model identified for the Carreau law enriched temperature is almost equivalent to the power law model. Comparison between the experimental datas and the identified Carreau law for the neat PP and each PP/MWCNT composite is related in the Figure 4. One can notice a good correlation between experimental and continuous datas.

\section{Conclusion}

The determination of the rheological properties of
PP/MWCNT with various MWCNT contents estimated in the temperature range from $180^{\circ} \mathrm{C}$ to $240^{\circ} \mathrm{C}$ has been leaded to set up and to build a constitutive model for the flow of PP/MWCNT based on the extension of the wellknown Carreau law coupled with a thermal factor. The resulting parameters have been identified for the neat PP and the PP/MWCNT composites. This constitutive model could be used to predict the PP/MWCNT composite flow of the in many process. In the future it will be interesting to consider a microscopic two-phase model to predict the CNT localization during one process. This study will be the subject of future investigations.

\section{REFERENCES}

[1] S. Iijima, "Helical Microtubes of Graphitic Carbon,” $\mathrm{Na}$ ture, Vol. 354, No. 6348, 1991, pp. 56-58. doi:10.1038/354056a0

[2] N. Coleman, U. Kahn, W. J. Blau and Y. K. Gun'ko, "Small but Strong: A review of the Mechanical Properties of Carbon Nanotube-Polymer Composites," Carbon, Vol. 44, 2006, No. 9, pp. 1644-1652. doi:10.1016/j.carbon.2006.02.038

[3] O. Breuer and U. Sundaraj, "Big Returns from Small Fibers: A Review of Polymer/Carbon Nanotube Composites," Polymer Composites, Vol. 25, No. 6, 2004, pp. 630-645. doi:10.1002/pc. 20058

[4] C. C. Teng, C. C. M. Ma, Y. W. Huang, S. M. Yuen, C. C. Weng, C. H. Chen and S. F. Su, "Effect of MWCNT Content on Rheological and Dynamic Mechanical Properties of Multiwalled Carbon Nanotube/Polypropylene Composites," Composites Part A: Applied Science and Manufacturing, Vol. 39, No. 12, 2008, pp. 1869-1875. doi:10.1016/j.compositesa.2008.09.004

[5] M. K. Seo and S. J. Park, "Electrical Resistivity and Rheological Behaviors of Carbon Nanotubes-Filled Poly- 
propylene Composites," Chemical Physics Letters, Vol. 395, No. 1-3, 2004, pp. 44-48. doi:10.1016/j.cplett.2004.07.047

[6] M. M. Cross, "Rheology of Non-Newtoinan Fluids: A New Flow Equation for Pseudoplastic Systems,” Journal of Colloid Science, Vol. 20, No. 5, 1965, pp. 417-437. doi:10.1016/0095-8522(65)90022-X

[7] P. Pötschke, A. R. Bhattacharyya, A. Janke and H. Goering, "Melt Mixing of Polycarbonate/Multi-Wall Carbon Nanotube Composites," Composite Interferences, Vol. 10, No. 45, 2003, pp. 389-404. doi:10.1163/156855403771953650

[8] S. H. Lee, M. W. Kim, S. H. Kim and J. R. Youn, "Rheological and Electrical Properties of Polypropy-
lene/MWCNT Composites Prepared with MWCNT Masterbatch Chips,” European Polymer Journal, Vol. 44, No. 6, 2008, pp. 1620-1630. doi:10.1016/j.eurpolymj.2008.03.017

[9] Y. C. Lam, Z. Y. Wang and S. C. Joshi, "Wall Slip of Concentrated Suspension in Capillary Flows," Powder Technology, Vol. 177, No. 3, 2007, pp. 162-169. doi:10.1016/j.powtec.2007.03.044

[10] P. J. Carreau, "Rheological Equations from Molecular Network Theories,” Journal of Rheology, Vol. 16, No. 1, 1972, pp. 99-127. doi:10.1122/1.549276 